Pacific Journal of Mathematics

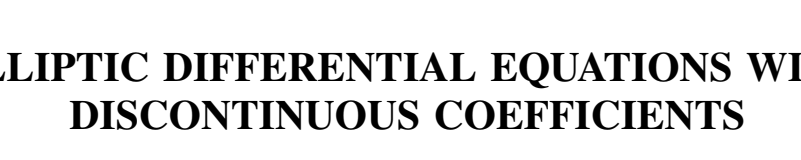




\title{
ELLIPTIC DIFFERENTIAL EQUATIONS WITH DISCONTINUOUS COEFFICIENTS
}

\author{
D. P. SQUiER
}

\begin{abstract}
The purpose of this paper is to furnish a proof of the following theorem:
\end{abstract}

Theorem. $D_{1}$ and $D_{2}$ are two disjoint open sets in the $x y$ plane having the open arc $\sigma$ as a common boundary. $L_{\imath}$ in $D_{i}, i=1,2$, are defined as

$$
\begin{aligned}
L_{i}(\phi) \equiv & a_{i} \phi_{x x}+2 b_{\imath} \phi_{x y}+c_{i} \phi_{y y}+d_{i} \phi_{x} \\
& +e_{i} \phi_{y}+g_{i} \phi, a_{\imath} c_{i}-b_{i}^{2}>0 .
\end{aligned}
$$

Functions $u_{i}$ satisfy $L_{i}\left(u_{i}\right)=f_{i}$ in $D_{i}$, with $u_{i} \in C^{2}$ in $D_{\imath}$ and $\in C^{1}$ in $D_{\imath} U_{\sigma}$; on $\sigma, u_{1}=u_{2}$ and $\partial u_{1} / \partial N_{1}=k(s) \partial u_{2} / \partial N_{2}$, where $s$ is arc length on $\sigma, k(s)>0$, and $\partial u_{i} / \partial N_{i}$ denotes the conormal derivative of $u_{\imath}$. If, on $D_{i} U \sigma, a_{i}, b_{i}, c_{i} \in C_{\alpha}^{n+2} ; d_{i}, e_{i}, g_{i}, f_{i} \in C_{\alpha}^{n}$; $k \in C_{\alpha}^{n+2}$ and $\sigma \in C_{\alpha}^{n+3}$; then $u_{i} \in C^{n+2}$ on $D_{i} U_{\sigma}$ for $n \geqq 0$. If all indicated quantities are analytic functions of their arguments and $\sigma$ is an analytic arc, then $u_{i}$ is analytic on $D_{i} U \sigma$.

Here $u \in C_{\alpha}^{n}$ on $G$ means the $n$th order derivatives of $u$ satisfy a uniform Hölder condition with exponent $\alpha$ on every compact subset of $G$. The conormal derivative

$$
\partial u_{i} / \partial N_{i}=\left(a_{i} r+b_{i} s\right) u_{x}+\left(b_{i} r+c_{i} s\right) u_{y}, r^{2}+s^{2}=1,
$$

uses the same unit normal $(r, s)$ to $\sigma$ for $i=1$ or 2 ; this normal may point into $D_{1}$ or it may point into $D_{2}$.

Such elliptic equations occur in physical problems involving continuous media of different properties. Smoothness of solutions plays an important role in the numerical analysis of such problems [7], [6].

Oleinik [5] has investigated the smoothness of solutions to such problems in several dimensions starting with weaker differentiability hypotheses on the $u_{i}$. This work differs from hers in that here analyticity is proved, and no restriction on $g_{i}$ is required for uniqueness of solutions is not required in the proof. The proof here is also along different lines since the restriction to two dimensions allows the use of conformal mapping and the Beltrami differential equation to bring $L_{i}$ to normal form, allowing previous results of the author [8] to be applied.

2. Since the proof of the theorem is based on coordinate transformations it is essential to examine how the various coefficients and the problem as a whole are altered under point transformations. With the symbolism 


$$
\begin{array}{ll}
\nabla_{x y}=\left(\begin{array}{c}
\frac{\partial}{\partial x} \\
\frac{\partial}{\partial y}
\end{array}\right), \quad J=\left(\begin{array}{ll}
\phi_{x} & \psi_{x} \\
\phi_{y} & \psi_{y}
\end{array}\right), \quad H=\left(\begin{array}{ll}
a & b \\
b & c
\end{array}\right), \\
\nabla_{x y}=J \nabla_{\xi \eta}, \quad J \text { nonsingular }, \quad A^{T} \equiv \text { transpose of } A,
\end{array}
$$

the second-order terms (principal part) in

$$
\left(\nabla_{x y}^{T} H \nabla_{x y}\right) u
$$

give $a u_{x x}+2 b u_{x y}+c u_{y y}$. Under the transformation $\xi=\phi(x, y), \eta=$ $\psi(x, y)$

$$
\nabla_{x y}^{T} H \nabla_{x y}=\left(J \nabla_{\xi \eta}\right)^{T} H J \nabla_{\xi \eta} .
$$

Thus the principal part of the right side in $\xi-\eta$ coordinates is associated with the matrix $J^{T} H J$. An arc $\sigma$ in the $x y$-plane $h(x, y)=0$ become $h^{*}(\varepsilon, \eta)=0$ and the normals $\nabla_{x y} h$ and $\nabla_{\xi \eta} h^{*}$ satisfy

$$
\nabla_{x y} h=J \nabla_{\xi \eta} h^{*} \text {. }
$$

Thus the conormal direction in the $x y$-plane

$$
\frac{1}{\|\nabla h\|} H \nabla h=N
$$

becomes in the $\xi \eta$ plane

$$
N^{*}=\frac{1}{\left\|\nabla h^{*}\right\|} J^{T} H J \nabla h^{*}=\frac{1}{\left\|J^{-1} \nabla h\right\|} J^{T} H \nabla h .
$$

And the conormal derivative $N^{T} \nabla_{x y} u$ becomes $\left(N^{*}\right)^{T} \nabla_{\xi \eta} u$ with

$$
\begin{aligned}
\left(N^{*}\right)^{T} \nabla_{\xi \eta} u & =\frac{1}{\left\|J^{-1} \nabla h\right\|}(\nabla h)^{T} H^{T} J J^{-1} \nabla_{x y} u \\
& =\frac{\|\nabla h\|}{\left\|J^{-1} \nabla h\right\|} N^{T} \nabla_{x y} u
\end{aligned}
$$

where \|\| is the usual Euclidean length of a vector.

Under a transformation of class $C_{\alpha}^{n}$ with nonvanishing Jacobian an arc of class $C_{\alpha}^{n}$ is transformed into an arc of class $C_{\alpha}^{n}$ and corresponding arc length parameters are connected by an invertible transformation of class $C_{\alpha}^{n}$, if $n \geqq 1$.

Thus in the theorem, if $D_{1} U D_{2} U \sigma$ is subjected to a (nonsingular) transformation of class $C_{\alpha}^{n+3}$, the problem transforms into a problem of the same type. A similar statement holds in the analytic case if the transformation is analytic.

The plan of proof is to map $\sigma$ into the $x$-axis and the principal 
parts of $L_{i}$ into Laplacian operators, then apply the results of [8]. In [8], the theorem has been proved for Laplacian principal parts for $k$ constant, but the proof carries through for $k$ variable, though the second derivatives of $k$ will appear in the coefficients of first order derivative terms of the modified equations.

The theorem is proved for a neighborhood of a point on $\sigma$; then a Heine-Borel argument extends the result to a neighborhood of $\sigma$.

3. If $\sigma$ is analytic and $p_{0}$ is a point on $\sigma$, there is a conformal map (nonsingular) taking $p_{0}$ into the origin and a neighborhood of $p_{0}$ onto a neighborhood of the origin in such a way that the points on $\sigma$ are mapped onto the $x$-axis. Since the mapping is one to one and analytic both ways, the problem becomes one of proving the analytic case of the theorem when $\sigma$ is the $x$-axis and $D_{1}$ is the region $y>0, D_{2}$ the region $y<0$.

As is known, $L(u)=a u_{x x}+2 b u_{x y}+c u_{y y}$ may be brought to a form with Laplacian principal part by a transformation $\xi=\phi(x, y)$ $\eta=\psi(x, y)$ if $\phi, \psi$ satisfy the Beltrami system

$$
\begin{aligned}
& \phi_{x}=\frac{b \psi_{x}+c \psi_{y}}{\delta} \\
& \phi_{y}=-\frac{a \psi_{x}+b \psi_{y}}{\delta} \\
& \delta^{2}=a c-b^{2}>0
\end{aligned}
$$

and are at least twice continuously differentiable. Indeed

$$
L(u)=\left(a \psi_{x}^{2}+2 b \psi_{x} \psi_{y}+c \psi_{y}^{2}\right)\left(\nabla_{\xi \eta}^{2} u\right)+\text { lower terms },
$$

where the lower terms involve the second derivatives of $\psi$ and first derivatives of $u$.

With $\delta_{i}^{2}=a_{i} c_{i}-b_{i}^{2}>0$ and the notation of the theorem, we solve the initial value problems

$$
\begin{aligned}
& \frac{\partial}{\partial x}\left(\frac{a_{i} \psi_{i x}+b_{i} \psi_{i y}}{\delta_{i}}\right)+\frac{\partial}{\partial y}\left(\frac{b_{i} \psi_{i x}+c_{i} \psi_{i y}}{\delta_{i}}\right)=0 \\
& \text { with } \psi_{i}(x, 0)=0, \psi_{i y}(x, 0)=\frac{\delta i}{c_{i}}, i=1,2 .
\end{aligned}
$$

Since $a_{i}, b_{i}, c_{i}, \delta_{i}$ are all analytic, (7) is an elliptic second order linear equation with analytic coefficients with prescribed analytic initial data. By the Cauchy-Kowaleski theorem there exists in a neighborhood of the $x$-axis a unique analytic $\psi_{i}$ satisfying the initial value problem. Both $\psi_{1}$ and $\psi_{2}$ are two-sided solutions since the $a_{i}$, etc., are analytic in a two-sided neighborhood of $\sigma$. From (7) it follows that the righthand side of (5) are components of the gradient of a scalar $\phi$, which may be constructed from the gradient by a line integral 


$$
\dot{\phi}_{i}(x, y)=\int_{(0,0)}^{(x, y)} \frac{b_{i} \psi_{i x}+c_{i} \psi_{i y}}{\delta_{i}} d x-\frac{a_{i} \psi_{i x}+b_{i} \psi_{i y}}{\delta_{i}} d y
$$

Since by (7) the integral is independent of path, the path for computing $\phi_{i}(x, 0)$ is the $x$-axis. Since $\psi_{i x}(x, 0)=0$ from the initial values, $\phi_{i}(x, 0)=x, i=1,2$.

The transformation $\xi=\dot{\phi}_{i}(x, y), \eta=\psi_{i}(x, y), x, y$ in $D_{i} U \sigma$ maps a neighborhood of the origin in the $x y$-plane onto a neighborhood of the origin in the $\xi \eta$-plane with the $x$-axis mapped onto the $\xi$-axis. The portion $y>0$ is mapped onto $\eta>0$; and $y<0$ onto $\eta<0$. The Jacobian

$$
\left|\begin{array}{cc}
\phi_{i x} & \dot{\phi}_{i y} \\
\psi_{i x} & \psi_{i y}
\end{array}\right|=\left|\begin{array}{cc}
\frac{c_{i}}{\delta_{i}} \psi_{i y} & \phi_{i y} \\
0 & \psi_{i y}^{\prime}
\end{array}\right|=\frac{\delta_{i}}{c_{i}}>0
$$

on $y=0$ and, therefore, the mapping in each region, analytically continuable into the other, is invertible, the inverse being analytic. The problem in the theorem is now reduced to one in which the equations have Laplacian principal parts (after division by $\left(a_{i} \psi_{i x}^{2}+\right.$ $2 b_{i} \psi_{i x} \psi_{i y}+c_{i} \psi_{i y}^{2}$ ) as indicated in (6) with $i=1$ for $\eta>0, i=2$ for $\eta<0$ ) and $\sigma$ is the $\xi$-axis. By [8], the solutions have the analyticity property on the $\xi$-axis, i.e., the solution in the region $\eta>0$ is analytically continuable into the lower region $\eta<0$ and conversely. Since every mapping from the original problem to this canonical problem has nonvanishing Jacobian on $\sigma$, the inverses are also analytic in a neighborhood of $p_{0}$ on $\sigma$ and, therefore, the solutions of the equations have the analyticity property on $\sigma$.

Since with each point on $\sigma$ there is a neighborhood of the point in which the solutions are analytic, it is possible to cover any closed subarc of $\sigma$ with a finite number of such neighborhoods which overlap. The solutions are analytic in the union of these neighborhoods.

4. In the nonanalytic case of the theorem, there is a mapping of class $C_{\alpha}^{n+3}$ with nonvanishing Jacobian taking $p_{0}$ on $\sigma$ into the origin and a neighborhood of $p_{0}$ into a neighborhood of the origin in such a way that $\sigma$ maps onto the $x$-axis with $D_{1}$ mapped into $y>0$ and $D_{2}$ into $y>0$. This is by definition of $\sigma \in C_{\alpha}^{n+3}$. Thus, as before, we may assume in the theorem that $\sigma$ is the $x$-axis. Again we use the Beltrami system to bring $L_{i}(u)$ into Laplacian principal part by solving (7). (7) is a linear second order elliptic equation with coefficients in $C_{\alpha}^{n+1}$. However, the Cauchy-Kowaleski theorem cannot be used to establish the existence of the desired mapping because the data is not analytic. Instead, the Schauder theory gives the existence. 
We let $K_{1}$ be a curve in $D_{1} U \sigma$ of class $C_{\alpha}^{n+3}$ coinciding with a segment of the $x$-axis containing the origin in the middle of the segment. From the Schauder theory there exists $\psi_{1}(x, y)$ of class $C_{\alpha}^{n+3}$ in the closed region bounded by $K_{1}$ satisfying (7) and assuming on $K_{1}$ the boundary values $\psi_{1}=y$. By Hopf's theorem [2], $\psi_{1 y}(x, 0)>0$. The $\phi$ in (5) is again reconstructed from its gradient by a line integral as in (8), the curve being confined to the closure of the region bounded by $K_{1}$. The Jacobian of the transformation $\xi=\phi_{1}(x, y), \eta=\psi_{1}(x, y)$ at $x=0, y=0$ is

$$
\left|\begin{array}{ll}
\dot{\phi}_{1 x} & \dot{\phi}_{1 y} \\
\psi_{1 x} & \psi_{1 y}
\end{array}\right|=\dot{\phi}_{1 x} \dot{\psi}_{1 y}=\frac{c_{1}}{\delta_{1}} \psi_{1 y}^{2}>0
$$

The function pair $\phi_{1}, \psi_{1}$ can be extended into $D_{2}$ as class $C_{\alpha}^{n+3}$ functions [1]. (This can easily be done for $x \in K_{1} \cap \sigma$. If

$$
h(x, y)=\sum_{j=0}^{n+3} \frac{\partial^{j} \psi_{1}(x, 0)}{\partial y^{j}} \frac{y^{j}}{j !}
$$

then $v(x, y)=\psi_{1}(x, y)-h(x, y)$ vanishes with its $y$ derivatives of order $\leqq n+3$ for $y=0$. Thus $v$ defined in $D_{1}$ is easily extended into $D_{2}$ by $v(x,-y)=v(x, y)$. If $V(x, y)$ is the extended $v, V \in C_{c}^{n+3}$ in $D_{1} U \sigma U D_{2}$. $\psi(x, y)=V(x, y)+h(x, y)$ is therefore the desired extension of $\psi_{1}$. $)$ Therefore, there is a class $C_{\alpha}^{n+3}$ transformation mapping a neighborhood of the origin in such a way that the $x$-axis goes into the $\xi$-axis, $L_{1}$ transforms into an operator which has principal part equal to a (nonvanishing) scalar times the Laplacian, as in (6), and the Jacobian does not vanish in the neighborhood. $L_{2}$ is also transformed into a linear second order elliptic operator. Both new equations are now divided by the coefficient of the Laplacian. Thus the theorem is now reduced to the case in which $L_{1}$ is the Laplacian and $\sigma$ is the $x$-axis.

We now use the Beltrami system to bring the current $L_{2}$ to Laplacian principal part, proceeding initially as above to obtain $\phi_{2}(x, y)$ and $\psi_{2}(x, y)$ in $D_{2} U \sigma$ such that $\psi_{2}(x, 0)=0$. Then the following transformation is applied to a neighborhood of the origin:

$$
\left.\left.\begin{array}{l}
\xi=\phi_{2}(x, y) \\
\eta=\psi_{2}(x, y)
\end{array}\right\} y \leqq 0 ; \quad \begin{array}{l}
\xi=\dot{\phi}_{2}(x, 0) \\
\eta=y
\end{array}\right\} y \geqq 0 .
$$

The mapping of $D_{1}$ has a $C_{\alpha}^{n+3}$ extension into $D_{2}$ and the mapping of $D_{2}$ has a $C_{\alpha}^{n+3}$ extension into $D_{1}$. Both mappings agree on $y=0$ and have nonvanishing Jacobian at $(0,0)$.

$$
\begin{aligned}
L_{1}\left(u_{1}\right)= & {\left[c_{2}(x, 0) \dot{\psi}_{2 y}(c, 0) / \delta_{2}(x, 0)\right]^{2} u_{1 \xi \xi}+u_{1 r \eta} } \\
& + \text { derivatives of } u_{1} \text { of lower order }
\end{aligned}
$$




$$
\begin{aligned}
L_{2}\left(u_{2}\right)= & \left(a_{2} \psi_{2 x}^{2}+2 b_{2} \psi_{2 x} \psi_{2 y}+c_{2} \psi_{2 y}^{2}\right)\left(\nabla_{\xi n}^{2} u_{2}\right) \\
& + \text { derivatives of } u_{2} \text { of lower order }
\end{aligned}
$$

Thus

$$
\begin{aligned}
& L_{1}\left(u_{1}\right) \equiv h^{2}(\xi) u_{1 \xi \xi}+u_{1 \eta \eta}+\cdots \\
& L_{2}\left(u_{2}\right) \equiv g(\xi, \eta) \nabla_{\xi \eta}^{2} u_{2}+\cdots
\end{aligned}
$$

where $h$ and $g$ are positive and $\epsilon C_{\alpha}^{n+2}$. On account of (9) and (4), the $k(\xi)$ for $(10)$ is still of class $C_{\alpha}^{n+2}$. (It is at this point that $C_{\alpha}^{n+3}$ transformations are required, rather than $C_{\alpha}^{n+2}$ because of the degrading of the differentiability of $k$ at this step by introducing factors depending on the first derivatives of the transformation.) Division of the upper equation in (10) by $h^{2}(\xi)$ and the lower one by $g(\xi, \eta)$ yields

$$
\begin{aligned}
& L_{1}\left(u_{1}\right)=u_{1 \xi \xi}+q^{2}(\xi) u_{1 \eta \eta}+\cdots \\
& L_{2}\left(u_{2}\right)=\nabla_{\xi \eta}^{2} u_{2}+\cdots
\end{aligned}
$$

the dots indicating terms involving $u$ derivatives of order less than two. The $k(\xi)$ for (11) is just the $k$ for (10) multiplied by $g(\xi, 0) / h^{2}(\xi)$. Thus the theorem has been reduced to the case (11) with $\sigma$ the $\xi$-axis.

Returning to $x-y$ variables to avoid excessive notation we make the variable change $x=\xi, y=\eta / q(\xi)$ for $\eta \geqq 0, x=\xi, y=\eta$ for $\eta \leqq 0$. This leaves $L_{2}$ unchanged, and brings $L_{1}$ into

$$
L_{1}\left(u_{1}\right)=u_{1 x x}+2 r(x) y u_{1 x y}+\left(r^{2} y^{2}+1\right) u_{1 y y}+\cdots
$$

where $r(x)=-q^{\prime} / q$. Thus the coefficients in the principal part of $L_{1}$ meet with those of $L_{2}$ to make them Lipschitz continuous throughout $C(0, a)$, a circle in $D_{1} U \sigma U D_{2}$ centered at $(0,0)$ with radius $a$.

As in [8] we write $u_{1}=u+k w, u_{2}=u+w$ where $u$ is even, $w$ odd, and both $\in C^{1}$ in $C(0, a)$. This leads to an elliptic system as in [8], but somewhat more complicated. However, the coefficients of second order derivative terms are Lipschitz continuous, and as in (12), the $x$-derivative of those coefficients are Lipschitz in $C(0, a)$. By Theorem 4.3 in [3], $u, w \in C_{\alpha}^{1}$ in $C(0, a)$. Now the proof of Theorem 4.5 in [3] can be applied, modified to use only derivatives and difference quotients in the $x$-direction, noting that the $v$ in that proof is of class $C_{\alpha}^{1}$ with one even component, one odd, but that in any case $v_{x} \in C_{\alpha}^{1}$. That proof puts $u_{x}, w_{x}$ in $C_{\alpha}^{1}$ in $C(0, a) . \quad u_{y y}$ and $w_{y y}$, for $y \geqq 0$, are expressed in terms of quantities which are of class $C_{\alpha}$ from the differential equations. Thus, for $y \geqq 0, u$ and $w$ are of class $C_{\alpha}^{2}$.

The further differentiability properties on $u$ and $w$ are obtained by differentiating the equations with respect to $x$ (using always the known interior differentiability properties) and observing that $u_{x}, w_{x}$ satisfy an elliptic system like $u$ and $w$ with the same boundary con- 
ditions on $y=0$. Thus $u_{x}, w_{x} \in C_{\alpha}^{2}$ for $y \geqq 0$. The equations for $y \geqq 0$ are then differentiated with respect to $y$ and solved for $u_{y y y}$ and $w_{y y y}$ in terms of quantities which are class $C_{\alpha}$. The process may be repeated as often as is possible to differentiate the coefficients. The differentiability properties of $u_{i}$ then follow.

This result gives three orders of differentiability more than the results in [5].

5. The theorem is valid if the solution pair $u_{1}, u_{2}$ are initially known only to be in $H_{2}^{1}$. (Classes $H_{p}^{m}$ are defined in [4], pp. 62-63.) By this, here, is meant that for any closed subset $S^{*}$ of $S=D_{1} U \sigma U D_{2}$ there is a sequence of function pairs $\left(u_{1}^{j}, u_{2}^{j}\right), u_{i}^{j} \in C^{1}$ in $D_{i} U \sigma$, satisfying the conormal derivative condition of the theorem, such that $u_{i}^{j}$ converges in the mean of order two to $u_{i}$ on $S^{*} \cap D_{i}$ while the first order derivatives form a Cauchy sequence in mean of order two on $S^{*} \cap D_{i}$.

The proof will be merely sketched for the analytic case. The nonanalytic case is similar. Clearly the $H_{2}^{2}$ hypotheses hold for the transformed problem, so it is sufficient to prove the result for the canonical form above, as in [8]. As there, $u_{2}=u+v, u_{1}=u+k v$ where $u$ is even, $v$ is odd; and $u, v$ satisfy in $S$, which may be taken as the open circle $C(0, a)$ centered at $(0,0)$ with radius $a$,

$$
\int_{R^{*}} \frac{\partial u}{\partial n} d s=\int_{R} F_{1} d x d y, \quad \int_{R^{*}} \frac{\partial v}{\partial n} d s=\int_{R} F_{2} d x d y
$$

for almost all closed cells $R$ in $C(0, a)$ [3]. Here $F_{1}$ and $F_{2}$ are linear combinations of $u, v, f_{1}, f_{2}$, and the first order derivatives of $u$ and $v$ as in [8] with bounded coefficients. By Theorem 4.3 in [3], $u$ and $v$ are of class $C_{\alpha}^{1}$ in $C(0, a)$. Thus $u_{i}$ is of class $C_{\alpha}^{1}$ on $D_{i} U \sigma$. The further results then follow.

\section{REFERENCES}

1. M. R. Hestenes, Extension of the range of a differentiable function, Duke Math. J. 8 (1941), 183-192.

2. E. Hopf, A remark on linear elliptic differential equations of second order, Proc. Amer. Math. Soc. 3 (1952), 791-793.

3. C. B. Morrey, Second order elliptic systems of differential equations, Ann. of Math. Studies 33, Princeton Univ., 1954, 101-159.

4. C. B. Morrey, Multiple Integrals in the Calcuius of Variations, Springer-Verlag, New York, 1966.

5. O. A. Oleinik, Boundary value problems for linear equations of elliptic parabolic type with discontinuous coefficients, Amer. Math. Soc. Translations (2) 42, 175-194.

6. J. W. Sheldon, Algebraic approximations for Laplace's equation, MTAC, 12 (1958), 174-186.

7. J. Sheldon and D. Squier, Remarks on the order of convergence of discrete analogs for second-order elliptic equations, SIAM Review 4 (1962), 366-378. 
8. D. P. Squier, Regularity properties at interfaces of solutions of elliptic equations, Contributions to Differential Equations 1 (1963), 453-459.

Received August 19, 1968.

Colorado State University 


\section{PACIFIC JOURNAL OF MATHEMATICS}

\section{EDITORS}

H. ROYDEN

Stanford University

Stanford, California

Richard Pierce

University of Washington

Seattle, Washington 98105
J. DugundJI

Department of Mathematics

University of Southern California

Los Angeles, California 90007

BASIL GORDON

University of California

Los Angeles, California 90024

\section{ASSOCIATE EDITORS}

E. F. BECKENBACH

B. H. NeUmanN

F. WOLF

K. YOSHIDA

\section{SUPPORTING INSTITUTIONS}

UNIVERSITY OF BRITISH COLUMBIA

CALIFORNIA INSTITUTE OF TECHNOLOGY

UNIVERSITY OF CALIFORNIA

MONTANA STATE UNIVERSITY

UNIVERSITY OF NEVADA

NEW MEXICO STATE UNIVERSITY

OREGON STATE UNIVERSITY

UNIVERSITY OF OREGON

OSAKA UNIVERSITY

UNIVERSITY OF SOUTHERN CALIFORNIA
STANFORD UNIVERSITY

UNIVERSITY OF TOKYO

UNIVERSITY OF UTAH

WASHINGTON STATE UNIVERSITY

UNIVERSITY OF WASHINGTON

$* * \quad * \quad *$
AMERICAN MATHEMATICAL SOCIETY
CHEVRON RESEARCH CORPORATION
TRW SYSTEMS
NAVAL WEAPONS CENTER




\section{Pacific Journal of Mathematics}

Vol. 30, No. $1 \quad$ September, 1969

William Wells Adams, Simultaneous diophantine approximations and cubic irrationals ..................................... 1

Heinz Bauer and Herbert Stanley Bear, Jr., The part metric in convex

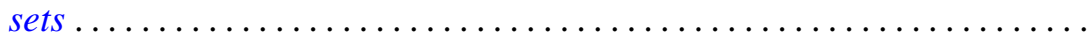

L. Carlitz, A note on exponential sums ...................... 35

Vasily Cateforis, On regular self-injective rings ................. 39

Franz Harpain and Maurice Sion, A representation theorem for measures on infinite dimensional spaces ......................... 47

Richard Earl Hodel, Sum theorems for topological spaces .............. 59

Carl Groos Jockusch, Jr. and Thomas Graham McLaughlin, Countable

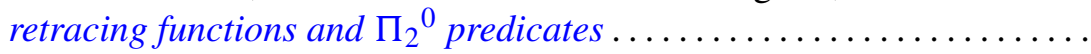

Bjarni Jónsson and George Stephen Monk, Representations of primary

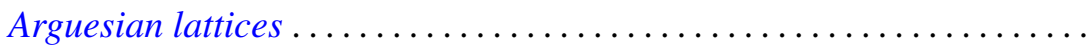

Virginia E. Walsh Knight, A continuous partial order for Peano continua...................................... 141

Kjeld Laursen, Ideal structure in generalized group algebras ........... 155

G. S. Monk, Desargues' law and the representation of primary lattices . . . 175

Hussain Sayid Nur, Singular perturbation of linear partial differential equation with constant coefficients ..........................

Richard Paul Osborne and J. L. Stern, Covering manifolds with cells ... 201

Keith Lowell Phillips and Mitchell Herbert Taibleson, Singular integrals in several variables over a local field...

James Reaves Smith, Local domains with topologically $T$-nilpotent radical....

Donald Platte Squier, Elliptic differential equations with discontinuous coefficients .................................

Tae-il Suh, Algebras formed by the Zorn vector matrix...

Earl J. Taft, Ideals in admissible algebras . .................... 259

Jun Tomiyama, On the tensor products of von Neumann algebras........ 263

David Bertram Wales, Uniqueness of the graph of a rank three group ..... 271

Charles Robert Warner and Robert James Whitley, A characterization of regular maximal ideals ......................... 